\title{
Exploring the characteristics of newly defined at-risk drinkers following the change to the UK low risk drinking guidelines: a retrospective analysis using Health Survey for England data
}

\author{
Philippa Case* (1), Linda Ng Fat and Nicola Shelton
}

\begin{abstract}
Background: Alcohol guidelines enable individuals to make informed choices about drinking and assist healthcare practitioners to identify and treat at-risk drinkers. The UK Low Risk Drinking Guidelines were revised in 2016 and the weekly guideline for men was reduced from 21 to 14 units per week. This study sought to retrospectively establish 1) the number of additional at-risk male drinkers in England, 2) which demographic characteristics were associated with being an at-risk drinker under the previous versus new guidelines.
\end{abstract}

Methods: Average weekly alcohol consumption for men aged 16+ from the cross-sectional nationally representative Health Survey for England were used to 1) calculate annual population prevalence estimates for newly defined at-risk ( $>14$ to $\leq 21$ units/week) male drinkers from 2011 to 2015 ( $N=3487-3790)$, and 2) conduct logistic regression analyses for at-risk vs low risk male drinkers under the previous ( $>21$ vs $\leq 21$ units/week) and new (> 14 vs $\leq 14$ units/week) guidelines to assess characteristics associated with being at-risk drinkers under each guideline using 2015 data ( $N=2982)$.

Results: Population prevalence estimates of newly defined at-risk drinkers ranged from 10.2\% (2014 =2,182,401 men)-11.2\% ( $2011=2,322,896$ men). Under the new guidelines, men aged 55-74 ( $\mathrm{OR}=1.63,95 \% \mathrm{Cl}=1.25-2.12)$; men in managerial/ professional occupations $(\mathrm{OR}=1.64,95 \% \mathrm{Cl}=1.34-2.00)$; current smokers $(\mathrm{OR}=2.26,95 \% \mathrm{Cl}=1.73-2.94)$, ex-regular smokers $(\mathrm{OR}=2.01,95 \% \mathrm{Cl}=1.63-2.47)$ and ex-occasional smokers $(\mathrm{OR}=1.85,95 \% \mathrm{Cl}=1.25-2.74)$; men from the North East (OR= $2.08,95 \% \mathrm{Cl}=1.38-3.13)$ and North West $(\mathrm{OR}=1.91,95 \% \mathrm{Cl}=1.41-2.60)$ of England all had greater odds, and non-white men had reduced odds $(\mathrm{OR}=0.53,95 \% \mathrm{Cl}=0.34-0.80)$ of being at-risk drinkers, as they had under the previous guidelines. Under the new guidelines only: a higher percentage of at-risk drinkers aged 16-34 (32\% vs 19\%) attenuated the odds of men aged $35-54$ being at-risk $(\mathrm{OR}=1.18,95 \% \mathrm{Cl}=0.92-1.51)$; a higher percentage of married at-risk drinkers (37\% vs $24 \%$ ) attenuated the odds of single men being at-risk ( $\mathrm{OR}=1.28,95 \% \mathrm{Cl}=0.99-1.67)$; men from the West Midlands ( $\mathrm{OR}=1.68,95 \% \mathrm{Cl}=1.17-$ 2.42) and London ( $\mathrm{OR}=1.53,95 \% \mathrm{Cl}=1.03-2.28)$ had greater odds of being at-risk drinkers.

Conclusions: The change to the Low Risk Drinking Guidelines would have resulted in more than 2 million additional male at-risk drinkers in England. Most groups with greater odds of being at-risk drinkers under the new guidelines were those already known to be drinking the most, strengthening the case for targeted screening and education. Additionally, under the new guidelines, a marked proportion of 16-35 year olds and married men were at-risk and men in the West Midlands and London had greater odds of being at-risk drinkers. These groups may benefit from specific education around the new Low Risk Drinking Guidelines.

Keywords: Alcohol, Drinking guidelines, Health survey for England, Alcohol risk, Sociodemographic characteristics, Alcohol screening

\footnotetext{
* Correspondence: philippa.case.17@ucl.ac.uk

Department of Epidemiology and Public Health, UCL 1-19 Torrington Place, London WC1E 7HB, UK
}

(c) The Author(s). 2019 Open Access This article is distributed under the terms of the Creative Commons Attribution 4.0 International License (http://creativecommons.org/licenses/by/4.0/) which permits unrestricted use, distribution, and reproduction in any medium, provided you give appropriate credit to the original author(s) and the source, provide a link to the Creative Commons license, and indicate if changes were made. The Creative Commons Public Domain Dedication waiver (http://creativecommons.org/publicdomain/zero/1.0/) applies to the data made available in this article, unless otherwise stated. 


\section{Background}

In England, alcohol guidelines form part of a suite of population level interventions to address problematic alcohol consumption [1,2]. They are used to aid individuals and healthcare professionals to assess the health risks associated with alcohol consumption [3, 4]. The UK Low Risk Drinking Guidelines were changed in 2016, reducing the maximum weekly alcohol consumption guideline down from 21 to 14 units per week for men, due to new evidence of the health risks associated with drinking at lower levels [3]. To assist with targeted screening for alcohol risk in healthcare services and targeted alcohol education programmes, this study used annual, nationally representative survey data to retrospectively explore how many men would have been redefined from low risk to at-risk drinkers according to the new guidelines, which demographic characteristics were associated with being an at-risk drinker before and after the change in guidelines and whether the characteristics associated with being an at-risk drinker changed under the new guidelines.

Globally, alcohol causes approximately $5.9 \%$ of deaths each year and is linked to over 200 disease and injury conditions, accounting for $5.1 \%$ of the global burden of disease [5] and presenting a significant public health risk. In England in 2016, over 23,000 deaths were alcohol-related [6] and despite a gradual decrease in the reported alcohol consumption of men in England [7], the number of hospital admissions linked to alcohol consumption in England continues to rise [8]. In addition to health consequences for the drinker, alcohol is linked to numerous other consequences such as violence and crime $[9,10]$, child abuse and neglect $[11,12]$, reduced productivity and unemployment $[13,14]$ and many more intangible consequences such as reduced quality of life, pain and suffering, both for the drinker and those around them [15].

In response to concerns over alcohol-related deaths and illnesses, the first UK guidelines on individual drinking were produced in 1984 in That's the Limit, with 'safe limits' described as 18 standard drinks (equivalent to 18 units) for men and 9 standard drinks for women [16]. Alcohol units (where 1 unit $=8 \mathrm{~g}$ pure alcohol) were introduced three years later along with a 'sensible limits' guideline of 21 units per week for men and 14 units per week for women, with 'too much' defined as 36 and 22 units respectively [16]. These guidelines continued until 1995, when, despite the British Medical Association [17] and the Royal Colleges of Physicians, Psychiatrists and General Practitioners [18] endorsing the continuation of the 21/14 units per week guideline, the Sensible Drinking guidelines changed to not regularly drinking more than 3 to 4 units per day for men and 2 to 3 units per day for women [19]. Whilst the move to daily limits caused concern amongst critics who viewed the change as, at best, being irrelevant and therefore ignored by the majority of drinkers who drink up to twice per week [20, 21 ] and at worst, encouraging daily drinking [22], all whilst significantly increasing the weekly limit [23]; in reality, the $21 / 14$ units per week message has persisted alongside the daily guideline. This was demonstrated following the introduction of the latest guidelines in 2016, recommending that men and women drink no more than 14 units per week [3], when numerous headlines, including one from the Department of Health [24] reported a reduction in the weekly guidelines for men from 21 to 14 units $[25,26]$.

The latest guideline review was prompted by new evidence (since the 1995 guidelines) regarding the health risks associated with alcohol consumption, particularly related to cancer risks, where the risk of developing certain cancers (including, but not limited to cancers of the throat, mouth and breast) is now known to increase even at low levels of alcohol consumption [20]. The new guidelines were developed following a consultation which reviewed the recent evidence [27] and used the Sheffield Alcohol Policy Model to review morbidity and mortality risk estimates related to different alcohol consumption levels [28]. The resulting Low Risk Drinking Guidelines state that, for both men and women: "To keep health risks from alcohol to a low level it is safest not to drink more than 14 units per week on a regular basis" (3, p4) with additional guidance around spreading these units across three or more days. The decision to align the male and female weekly guideline was taken following evidence that at lower levels of alcohol consumption, the risks for men and women are similar, with men being more vulnerable to shorter-term risks and women more vulnerable to longer-term risks in general [3].

The aim of drinking guidelines is to inform the public in order for people to make informed decisions about their alcohol consumption [3, 29]. Whilst drinking guidelines have been criticised for their ineffectiveness in reducing alcohol consumption or changing drinking behaviour [30,31], the value of drinking guidelines as part of a suite of education and information interventions, which also include health warnings on alcohol containers, counter-advertising and school interventions, has been recognised $[1,30]$. Research into public awareness of changes to alcohol guidelines in the UK has repeatedly found that awareness peaks around the time of the change but is not sustained and long-term promotional campaigns have been recommended [32-34].

In addition to informing the public, drinking guidelines enable healthcare practitioners to assess and advise patients on their alcohol consumption. Screening for at- 
risk drinkers (usually either defined as drinking above national guidelines or identified via alcohol risk screening tools such as the Alcohol Use Disorders Identification Toolkit (AUDIT) [35]) and offering brief interventions for alcohol use, such as brief structured advice or brief motivational interviewing [36] have demonstrated positive results [37]. Whilst statistical modelling to predict population-level benefits of screening and brief interventions support universal screening [36], National Institute for Health and Care Excellence (NICE) guidance recommends universal screening if feasible and targeted screening of groups at greater risk of alcohol-related harm (e.g. people with relevant physical or mental health problems) if universal screening is not feasible [4]. Widespread implementation of screening has proved problematic both in trials of screening and brief intervention [38] and in clinical practice [39], and policy in England has favoured targeted screening in general practice settings, including the incentivisation of primary care services in England between 2008 and 2015 to deliver 'Identification and Brief Advice' for new patients only [36].

With an increasing focus within healthcare on targeting at-risk drinkers who consume above the low risk drinking guidelines [4, 37-39], the recent reduction in the low risk drinking threshold for men from 21 to 14 units per week could have a significant impact on healthcare providers as the number of at-risk drinkers increases. Furthermore, new awareness of the health risks associated with consuming above 14 units of alcohol per week means that supporting people to reduce their consumption to within these levels is a public health priority. Establishing the number of additional men who would be classified as at-risk following the guideline change may assist policy and commissioning decisions relating to screening for at-risk drinkers and provide a further incentive for improving education and awareness around drinking guidelines. Furthermore, within the English policy model of targeted screening for at-risk drinkers, it would be of benefit to know which demographic characteristics are associated with being an at-risk drinker and to establish whether the change in guidelines affected any specific demographic groups. It is feasible that men who were previously adhering to the guidelines may a) be more amenable to adhering to recommended guidelines having previously consumed within the low risk guidelines and b) not be aware that they are no longer considered to be drinking at low risk levels; therefore this group may benefit from specific targeting. Consequently, the objectives of this study were:

1. To establish the number of additional male at-risk drinkers in England over the past 5 years according to the new Low Risk Drinking Guidelines.
2. To establish which demographic characteristics in men were associated with being at-risk ( $>21$ units/ week) vs low risk ( $\leq 21$ units/week) drinkers according to the previous guidelines; which characteristics were associated with being at-risk ( $>14$ units/week) vs low risk ( $\leq 14$ units/week) drinkers according to the new (2016) guidelines, and to explore whether the characteristics associated with being an at-risk drinker were different following the change to guidelines.

Whilst it could be argued that, in line with the 1995 Sensible Drinking guidelines of no more than 3 to 4 units per day for men [19], the change to guidelines constituted a $50 \%$ decrease from (a potential) 28 units per week to 14 ; both the media (e.g. 22-24), and the medical community $[17,18,23,40]$, including those defining increasing risk drinking in order to facilitate brief interventions in healthcare settings [4] viewed this as a reduction of one third in light of the pre-existing weekly limit of 21 units for men. Therefore, this paper will focus on a change in the Low Risk Drinking Guidelines from 21 units per week to 14 units per week for men.

\section{Methods}

The Health Survey for England (HSE) is an annual, nationally representative, cross-sectional survey using a clustered, stratified multi-stage sampling design to select a random sample of private households in England. The survey is administered face to face within the participant's household by a trained interviewer. Full details of the HSE methodology can be found online [41]. Ethical approval was sought prior to data collection from the relevant research ethics committee and further ethical approval was not required for this study.

\section{Measures}

Questions on alcohol consumption have been included in the HSE since it began in 1991. Participants from 2011 to 2015 were asked if they ever drink: 'Do you ever drink alcohol nowadays, including drinks you brew or make at home?' and for those participants who respond no, a follow up question was asked: 'Could I just check, does that mean you never have an alcoholic drink nowadays, or do you have an alcoholic drink very occasionally, perhaps for medicinal purposes or on special occasions like Christmas and New Year?'. For those who responded that they did drink alcohol, participants were asked about each type of alcohol in turn (e.g. normal strength beer, lager, stout, cider or shandy under $6 \%$ $\mathrm{ABV}$; wine, including Babycham and champagne etc.) and were shown a card which demonstrates the types of drink and the different sizes of measure. Participants were asked how often they had consumed that beverage 
during the past 12 months (1. Almost every day, 2. Five or six days per week.... 7. Once or twice a year, 8. Not at all in the last 12 months) and then asked how much of that alcoholic drink they consumed on an average day when they were drinking it (one question for the size of measure and one for the number of drinks consumed). This information was then combined to calculate an average weekly consumption (the 'quantity-frequency' method) measured in units. Whilst quantity-frequency methods for collecting data on alcohol are common, the specific questions used in the HSE have been developed over time for this survey. The full HSE questionnaire (2015) can be accessed online [42] with the alcohol questions contained on pages 61 to 70 and show cards on pages 156 and 157. Further details on the background to the alcohol questions and how they have developed over time since the HSE began in 1991 can also be found online [43].

\section{Objective 1}

Using the average weekly alcohol consumption measure, men were regrouped into: 1) non-drinkers (no alcohol consumed in past 12 months); 2) low risk drinkers ( $\leq 14$ units per week); 3 ) newly defined at-risk drinkers (>14 to $\leq 21$ units per week) and 4) previously defined at-risk drinkers (> 21 units per week).

\section{Objective 2}

Using the average weekly consumption measure, men were regrouped into:

1) At-risk ( $>21$ units per week) vs low risk ( $\leq 21$ units per week) male drinkers according to the previous guidelines, and.

2) At-risk ( $>14$ units per week) vs low risk ( $\leq 14$ units per week) male drinkers according to the new (2016) guidelines.

A number of demographic characteristics were identified from the literature as potentially impacting upon alcohol consumption levels and the corresponding measures from the HSE were selected for the analysis to establish whether at-risk male drinkers were distinct from low risk male drinkers under both the previous and the new drinking guidelines and to establish whether the change in guidelines affected the demographic characteristics associated with being an at-risk drinker. Demographic characteristics that have been associated with differing levels of alcohol consumption and were included in the analysis are: age [43], grouped from 16 to 34, 35-54, 55-74 and 75+; social class [44, 45], grouped using the National Statistics Socio-Economic Classification (NS-SEC) categorising the employment status of the participant (managerial and professional, intermediate, routine and manual, not classified); marital status [46, 47] grouped as single, married/cohabiting, separated/divorced/ widowed; geographical region [6, 48-50], grouped by former Government Office Region; ethnicity [51, 52], regrouped into white and non-white groups due to small sample sizes in the non-white groups; smoking status [53], grouped by never smoker, ex-occasional smoker, ex-regular smoker, current smoker; and physical health $[54,55]$ measured as limiting long-lasting illness, non-limiting long-lasting illness, no long-lasting illness. The full HSE questionnaire (2015) can be accessed online [42].

\section{Sample}

\section{Objective 1}

Data were utilized from all adult males (aged 16 and over), including non-drinkers and drinkers, but excluding those participants who had missing data on the alcohol measure ( $N=121$ (2011), $N=85$ (2012), $N=135$ (2013), $N=101$ (2014), $N=223$ (2015)). Data for the years 2011 to 2015 were selected because 2011 is the first year that the quantity-frequency measure was reinstated in the survey and 2015 was the most recent available dataset at the time that the data were analysed. Annual data were analysed separately. Sample sizes can be found in Table 1.

\section{Objective 2}

Data from all adult (aged 16 and over) male participants from HSE 2015 who reported any alcohol consumption in the past 12 months were used, excluding those participants with missing data for the alcohol variable $(N=$ 223). Participants with missing data from the other variables in the model were also excluded in the final sample. In all variables, the number of missing values constituted equal to or less than $1 \%$ of the sample (age: $N=0$; NS-SEC: $N=31$ (1.0\%); marital status: $N=1(<$ $1 \%$ ); cigarette smoking status: $N=0$; limiting long-lasting illness: $N=4(<1 \%)$; ethnicity: $N=2(<1 \%)$; former Government Office Region: $N=0$ ). The final sample for each logistic regression model was 3258 with weighting applied ( $N=2982$ unweighted). Sample characteristics can be found in Table 2. The data used in the analysis are from 2015 (the most recent year of data at the time of the analysis). The focus of this paper was to estimate the number of men who would have been redefined as at-risk drinkers following the guidelines change and to explore the characteristics of at-risk drinkers under the previous vs the new guidelines, rather than to explore any effect of the change in guidelines itself; therefore using retrospective data was considered appropriate.

\section{Statistical analyses}

All data were analysed using the complex survey data procedures in Stata 15.0 and using the weighting variables provided in HSE to account for the clustered and stratified sampling. 


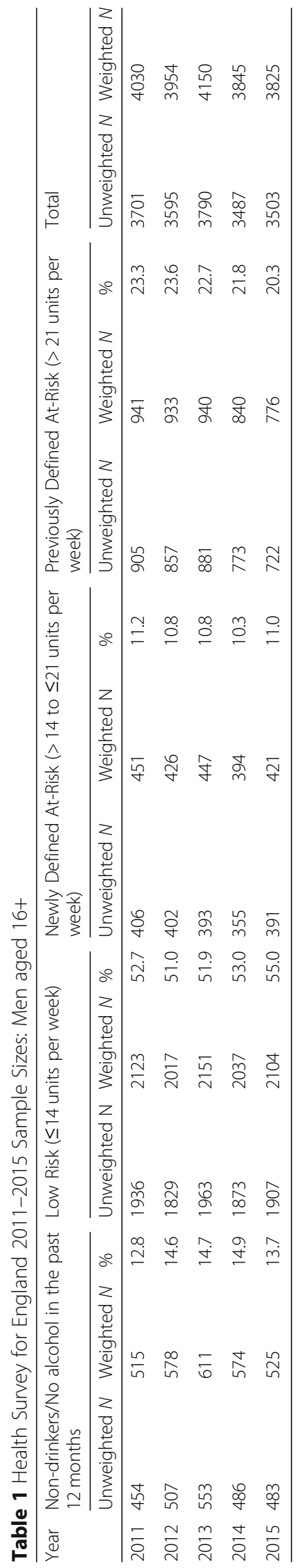




\section{Objective 1}

Annual population prevalence estimates for newly defined at-risk drinkers (men drinking $>14$ to $\leq 21$ units per week) were calculated for each age group, following the procedure described in the HSE technical annexe for calculating population estimates [56]. The total annual population prevalence was calculated by summing the age group totals.

\section{Objective 2}

Non-drinkers were removed from the data as the Low Risk Drinking Guidelines apply to drinkers only. Firstly, a logistic regression model was applied to assess which characteristics were significantly associated with men being previously defined at-risk drinkers vs low risk drinkers (reference group) (men drinking $>21$ vs $\leq 21$ units/week), then a second logistic regression model was applied to assess which characteristics were significantly associated with men being newly defined at-risk drinkers vs low risk drinkers (reference group) (men drinking > 14 vs $\leq 14$ units/week). The two models were then compared. As this was an exploratory analysis, all variables were entered into the model simultaneously.

\section{Results}

1. To establish the number of additional male at-risk drinkers in England over the past 5 years according to the new Low Risk Drinking Guidelines

Unweighted and weighted sample sizes are recorded in Table 1. Sample sizes were different in each year, ranging from 3487 to 3790 (unweighted). Whilst exact percentages varied from year to year, the newly defined at-risk group (men drinking $>14$ to $\leq 21$ units per week) was consistently the smallest group of men, followed by nondrinkers and previously defined at-risk drinkers (men drinking > 21 units per week), with low risk drinkers constituting the majority of the sample each year.

Table 3 shows the annual population prevalence estimates of adult men in England (2011-2015) drinking at newly defined at-risk levels ( $>14$ to $\leq 21$ units per week) by age group. The youngest (16-25 years) and oldest (75+ years) age groups consistently had amongst the lowest proportions of men drinking at newly defined atrisk levels, whilst the proportion of men drinking at newly defined at-risk levels in other age groups varied annually. The highest proportion in any age group categorised as newly defined at-risk drinkers was amongst 55 to 64-year-old men in 2015 (15.3\%).

The percentage of men aged 16 and over, living in private residences in England who were drinking at newly defined at-risk levels ranged from 11.2\% (2,322,896, 5.5\% total population) in 2011 to $10.2 \%(2,182,401$ men, $5 \%$ of the total population) in 2014 .
2. To establish which demographic characteristics in men were associated with being at-risk (> 21 units/week) vs low risk ( $\leq 21$ units/week) drinkers according to the previous guidelines; which demographic characteristics were associated with being at-risk (> 14 units/week) vs low risk ( $\leq 14$ units/week) drinkers according to the new (2016) guidelines, and to explore whether the characteristics associated with being an at-risk drinker were different following the change to guidelines.

Sample characteristics for low risk vs at-risk male drinkers under the previous guidelines (men drinking $\leq 21$ vs $>21$ units/week) and low risk vs at-risk male drinkers under the new guidelines (men drinking $\leq 14$ vs $>14$ units/week) taken from Health Survey for England 2015 data are shown in Table 2. In all age groups, a higher proportion of men were classified as at-risk drinkers under the new guidelines, in line with the lower weekly threshold. The difference in percentage of at-risk drinkers under the previous vs new guidelines was greatest for 55-74 year olds (29\% at-risk under the previous guidelines and 44\% at-risk under the new guidelines) followed by $16-34$ year olds (19\% under the previous guidelines and 32\% under the new guidelines).

Within the 2015 HSE sample, the demographic groups with the greatest difference in proportion of at-risk drinkers under the previous vs new guidelines were men working in managerial or professional occupations $(26 \%$ of men under the previous guidelines vs $41 \%$ under the new guidelines), married or cohabiting men (24\% vs $37 \%$ ), men living in the North East (30\% vs 46\%) and South West (22\% vs 38\%) of England, white men (25\% vs $38 \%$ ), men who were current (30\% vs $44 \%$ ) or exregular (29\% vs 43\%) smokers and men with nonlimiting long-lasting illness (24 vs $39 \%$ ). For all demographic variables, the groups with the highest percentage of at-risk drinkers remained similar under the new guidelines.

Table 4 shows the results of two logistic regression models: column 1 shows the odds ratios for at-risk vs low risk (reference category) drinkers under the previous guidelines ( $>21$ vs $\leq 21$ units/week) and column 2 shows the odds ratios for at-risk vs low risk (reference category) drinkers under the new guidelines ( $>14$ vs $\leq 14$ units/week). All results relate to data from the HSE 2015.

Most demographic characteristics which were significantly associated with at-risk drinking under the previous guidelines remained so under the new guidelines. Under both the previous (OR 1.71, 95\%CI 1.25-2.34) and new (OR 1.63, 95\%CI 1.25-2.12) guidelines, 55-74 year old men had significantly greater odds of being classified as at-risk (vs low risk) drinkers when compared to the youngest age group (16-34 year olds); the association was slightly attenuated by the lowered threshold 


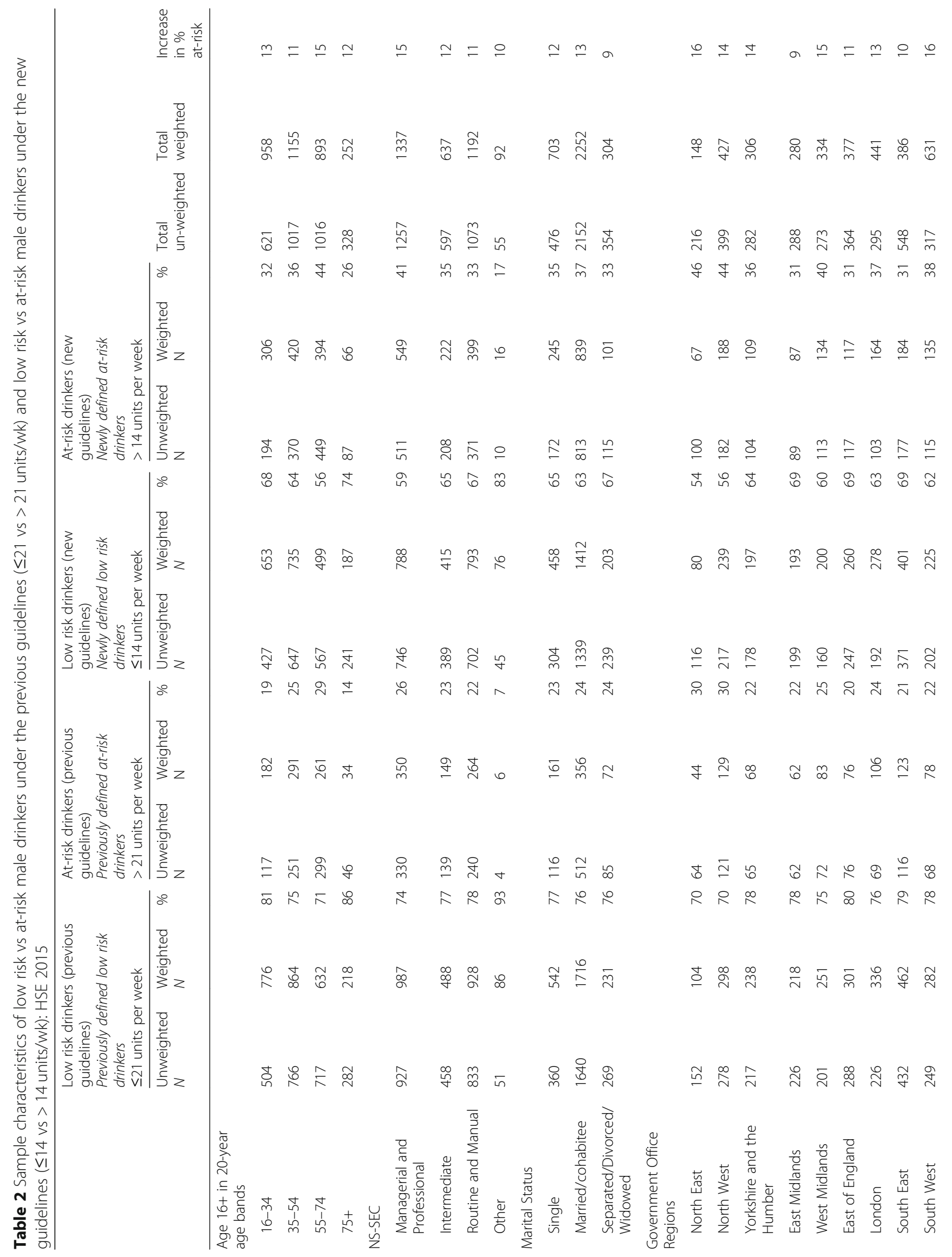




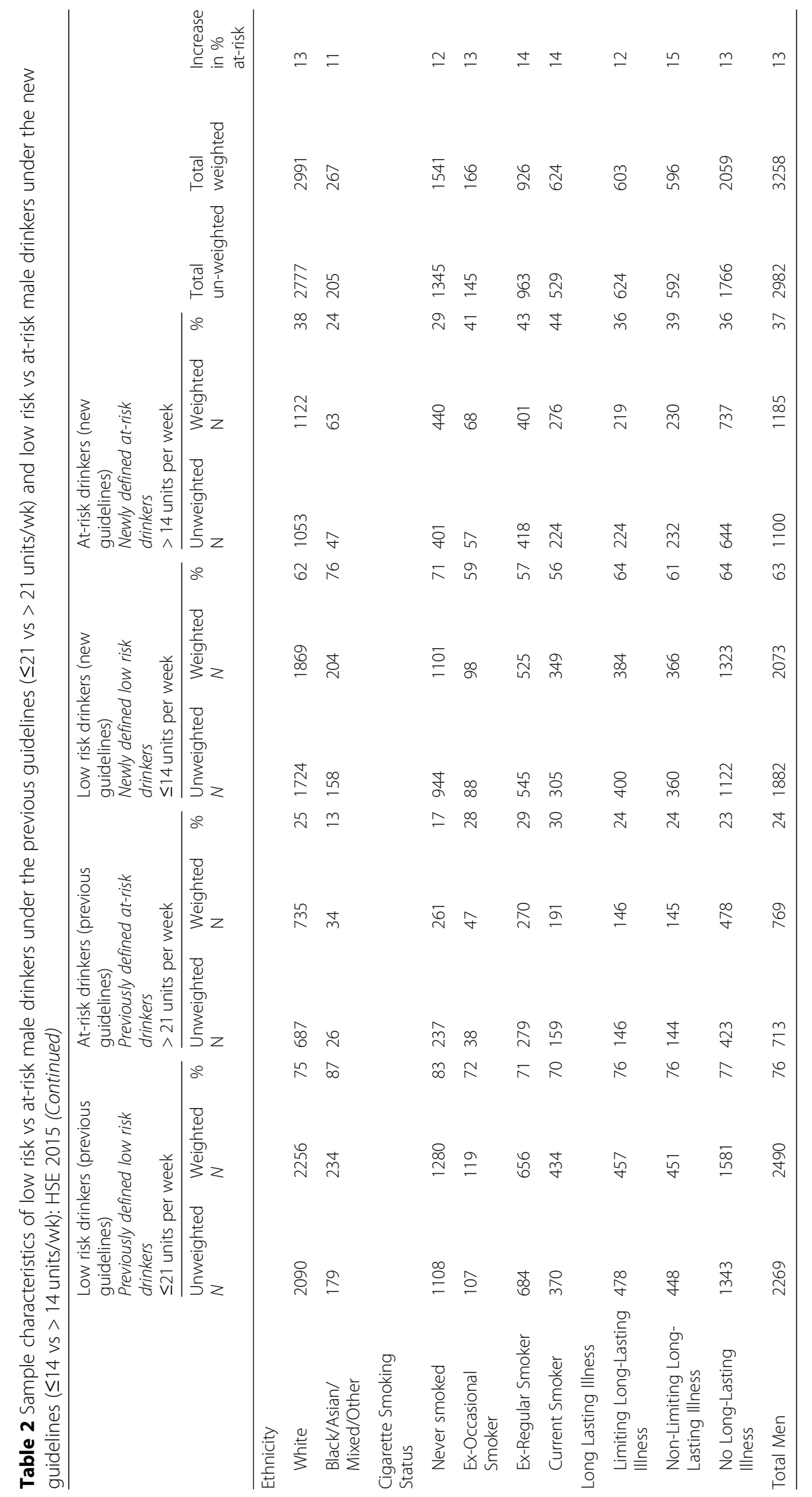


Table 3 Population estimates of men aged 16+ in England drinking at newly defined at-risk levels (> 14 to $\leq 21$ units per week) 2011-2015

\begin{tabular}{llllll}
\hline & \multicolumn{4}{l}{ Population Estimate for men drinking at newly defined at-risk (> 14 to $\leq 21$ units per week) } \\
\cline { 2 - 6 } Age group & 2011 & 2012 & 2013 & 2014 & 2015 \\
\hline $16-24$ & $267,202(8.4 \%)$ & $295,766(9.3 \%)$ & $303,767(9.6 \%)$ & $214,567(6.8 \%)$ & $202,012(6.4 \%)$ \\
$25-34$ & $451,190(12.6 \%)$ & $337,064(9.3 \%)$ & $452,814(12.3 \%)$ & $440,510(11.9 \%)$ & $498,080(13.3 \%)$ \\
$35-44$ & $511,584(13.9 \%)$ & $406,468(11.3 \%)$ & $395,989(11.1 \%)$ & $384,803(10.9 \%)$ & $337,036(9.5 \%)$ \\
$45-54$ & $385,854(10.7 \%)$ & $380,888(10.3 \%)$ & $395,096(10.6 \%)$ & $446,348(11.8 \%)$ & $395,929(10.4 \%)$ \\
$55-64$ & $394,857(13.0 \%)$ & $424,711(14.2 \%)$ & $306,709(10.3 \%)$ & $302,726(10.1 \%)$ & $467,067(15.3 \%)$ \\
$65-74$ & $184,154(8.5 \%)$ & $282,813(12.3 \%)$ & $265,744(11.1 \%)$ & $230,572(9.4 \%)$ & $289,205(11.5 \%)$ \\
$75+$ & $128,055(8.1 \%)$ & $134,078(8.2 \%)$ & $136,706(8.2 \%)$ & $162,875(9.3 \%)$ & $176,655(9.9 \%)$ \\
Total & $2,322,896$ & $2,261,788$ & $2,256,825$ & $2,182,401$ & $2,365,984$ \\
\% Male Population & 11.2 & 10.8 & 10.6 & 10.2 & 11.0 \\
\% Total Population & 5.5 & 5.3 & 5.2 & 5.0 & 5.4 \\
\hline
\end{tabular}

under the new guidelines. Under both the previous (OR $0.61,95 \%$ CI $0.40-0.92$ ) and new (OR 0.69, 95\% CI $0.48-0.98)$ guidelines, men over 75 years old had significantly lower odds of being at-risk (vs low risk) drinkers when compared to the youngest age group.

Under the previous guidelines, 35-54 year olds had significantly greater odds of being at-risk drinkers (vs low risk drinkers) compared to the youngest age group (OR 1.42, 95\% CI 1.08-1.98); however, under the new guidelines, 35-54 year old men no longer had significantly greater odds of being categorised as at-risk drinkers than the younger age group (OR 1.18, 95\% CI $0.92-1.51)$. This was also the case in the unadjusted regression model (OR 1.22, 95\% CI 0.97-1.54).

Men working in managerial or professional occupations had significantly greater odds of being classified as at-risk (vs low risk) drinkers compared to men working in manual or routine occupations under both the previous (OR 1.46, 95\% CI 1.15-1.86) and the new (OR 1.64, 95\% CI 1.34-2.00) guidelines, with the magnitude of association increasing following the change to the guidelines.

Under the previous guidelines, single men had significantly greater odds of being at-risk (vs low risk) drinkers compared to men who were married/cohabiting (OR 1.41, 95\% CI 1.06-1.88); however, under the new guidelines this association was no longer significant (OR 1.28, 95\% CI 0.99-1.67). It was also the case within the unadjusted regression model that single men did not have greater odds of being at-risk drinkers than married/cohabiting men under the new guidelines (OR 0.90, 95\% CI 0.71-1.13).

Under both the previous and new guidelines, men from the North East (previous: OR 1.75, 95\% CI 1.09_ 2.79; new: OR 2.08, 95\% CI 1.38-3.13) and North West (previous: OR 1.78, 95\% CI 1.26-2.51; new: OR 1.91, 95\% CI 1.41-2.60) of England had greater odds of being classified as at-risk (vs low risk) drinkers than men from the South East of England. Under the new (but not the previous) guidelines, men from the West Midlands (OR $1.68,95 \%$ CI 1.17-2.42) and London (OR 1.53, 95\% CI 1.03-2.28) had greater odds of being classified as at-risk drinkers than those in the South East.

For men in the West Midlands, this association was also significant in the unadjusted model (OR 1.46, 95\% CI 1.02-2.08). For men living in London, the association was not significant in the unadjusted model (OR 1.29, 95\% CI 0.87-1.91), and when all variables were entered in a stepped process, the association was significant only when ethnicity was also included in the model.

Under both the previous (OR 0.44, 95\% CI 0.25-0.77) and new (OR 0.53, 95\% CI 0.34-0.80) guidelines, nonwhite men had significantly lower odds of being at-risk drinkers than white men.

Current smokers (previous: OR 2.30, 95\% CI 1.723.08; new: OR 2.26, 95\% CI 1.73-2.94), ex-regular smokers (previous: OR 2.11, 95\% CI 1.67-2.67; new: OR 2.01, 95\% CI 1.63-2.47) and ex-occasional smokers (previous: OR 2.10, 95\%CI 1.36-3.25; new: OR 1.85, 95\% CI 1.25-2.74) all had significantly greater odds of being atrisk drinkers under both the previous and the new guidelines. The association was slightly attenuated under the new, lower guidelines.

Neither limiting, nor non-limiting long-lasting illness were significantly associated with being classified as an at-risk drinker, either under the previous or the new guidelines.

\section{Discussion}

The reduction in the Low Risk Drinking Guidelines for men from 21 to 14 units per week meant that between 2011 and 2015 over two million men each year, who would previously have been defined as drinking at low risk levels, would now be defined as at-risk drinkers. 
Table 4 Logistic Regression Models for at-risk vs low risk male drinkers under previous guidelines (> 21 vs $\leq 21$ units per week) and at-risk vs low risk male drinkers under the new guidelines (> 14 vs $\leq 14$ units per week) (HSE 2015)

Age 16+ in 20-year age bands

16-34

35-54

55-74

$75+$

NS SEC

Routine and Manual

Managerial and Professional

Intermediate

Other

Marital Status

Married/Cohabitee

Single

Divorced/Separated/Widowed

Government Office Regions

South East

North East

North West

Yorkshire and the Humber

East Midlands

West Midlands

East of England

London

South West

Ethnicity

White

Black/Asian/Mixed/Other

Cigarette Smoking Status

Never smoked

Ex-Occasional Smoker

Ex-Regular Smoker

Current Cigarette Smoker

Long-Lasting Illness

No Long-Lasting IIIness

Limiting Long-Lasting IIIness

Non-Limiting Long-Lasting IIIness

Constant

${ }^{*} P<0.05{ }^{* *} p<0.01{ }^{* * *} p<0.001$

These newly defined at-risk drinkers constituted an additional 11\% of the adult male population in England.

As health care services increasingly focus on screening for at-risk drinkers and offering brief interventions in
At-risk vs low risk under previous guidelines

(> 21 vs $\leq 21$ units per week) OR ( $95 \% \mathrm{Cl})$

At-risk vs low risk under new guidelines (> 14 vs $\leq 14$ units per week) OR (95\% Cl)
1.00
$1.42(1.08-1.89)^{*}$
$1.71(1.25-2.34)^{* *}$
$0.61(0.40-0.92)^{*}$

1.00

$1.46(1.15-1.86)^{* *}$

$1.16(0.86-1.55)$

$0.30(0.09-0.94)^{*}$

1.00

$1.41(1.06-1.88)^{*}$

$0.96(0.72-1.27)$

1.00

$1.75(1.09-2.79)^{*}$

$1.78(1.26-2.51)^{* *}$

$1.22(0.78-1.91)$

$1.14(0.81-1.60)$

$1.37(0.92-2.05)$

$1.04(0.69-1.55)$

$1.43(0.96-2.14)$

$1.04(0.72-1.51)$

1.00

$0.44(0.25-0.77)^{* *}$

1.00

$2.10(1.36-3.25)^{* *}$

$2.11(1.67-2.67)^{* * *}$

$2.30(1.72-3.08)^{* * *}$

1.00

$1.01(0.77-1.34)$

$0.99(0.76-1.28)$

$0.10(0.07-0.15)^{* * *}$
1.00

$1.18(0.92-1.51)$

$1.63(1.25-2.12)^{* * *}$

$0.69(0.48-0.98)^{*}$

1.00

$1.64(1.34-2.00)^{* * *}$

$1.14(0.88-1.49)$

$0.48(0.22-1.05)$

1.00

$1.28(0.99-1.67)$

$0.78(0.60-1.02)$

1.00

$2.08(1.38-3.13)^{* * *}$

$1.91(1.41-2.60)^{* * *}$

$1.42(0.97-2.08)$

$1.06(0.76-1.48)$

$1.68(1.17-2.42)^{* *}$

$1.09(0.75-1.61)$

$1.53(1.03-2.28)^{*}$

1.35 (0.97-1.88)

1.00

$0.53(0.34-0.80)^{* *}$

1.00

$1.85(1.25-2.74)^{* *}$

$2.01(1.63-2.47)^{* * *}$

1.00

$0.98(0.77-1.25)$

$1.05(0.85-1.30)$

$0.19(0.14-0.27)^{* * *}$
$2.26(1.73-2.94)^{* * *}$

order to reduce their alcohol intake [4, 37-39], the sudden increase in the number of at-risk drinkers by an additional 2.3 million men (based on 2015 figures) caused by the change to alcohol guidelines could have a 
significant impact on services, all at a time when incentivisation for identification and brief advice in primary care has been withdrawn [39]. Policy within England favours targeted screening [36]; a decision which is likely to have been influenced by the significant cost and practical implications of universal screening and the decision by the UK National Screening Programme not to recommend screening for alcohol misuse nationally [57]. It is therefore unlikely under the current system that these men will be identified through routine screening, and, particularly in light of the current evidence relating to poor awareness of changes to guidelines in the UK [3234], these men may well continue to drink at levels which pose a risk to their health.

Knowing which groups of men have greater odds of being at-risk drinkers, and particularly those groups which would have been redefined as at-risk drinkers following the change to guidelines, could allow for 1) more targeted screening for at-risk male drinkers, and 2) targeted and sustained educational programmes around drinking guidelines.

Under both the previous and the new drinking guidelines, men aged 55-74 had greater odds of being at-risk drinkers, which correlates with current evidence around 'middle-aged men' being the most at-risk in terms of their drinking [58] and reinforces the need for more targeted campaigns for this age group. This could also provide an age criterion with which to screen for at-risk drinkers. Under the previous, but not the new guidelines, men aged 35-54 had greater odds of being at-risk drinkers, a change which may be explained by the greater increase in the proportion of at-risk drinkers in the reference category (16-34 year olds, $13 \%$ increase in at-risk drinkers) compared to $35-54$ year olds $(11 \%$ increase in at-risk drinkers). Around a third of men in the younger age group would now be considered at-risk from their drinking and the youngest age group experienced the second largest increase in the number of people considered at-risk under the new guidelines, indicating that a considerable proportion of this age group were drinking between 14 and 21 units per week. With this in mind, it is important to ensure that any sustained promotional activity does not focus on one age group to the detriment of others as has been suggested with the former focus on problematic younger drinkers [59].

Further criteria for targeted screening and education might include white men and men working in managerial and professional occupations, who had consistently greater odds of being at-risk drinkers in line with existing evidence $[45,52]$. Routes to delivery here might be through screening and education in workplaces. The limited sample size only permitted analysis of a binary ethnicity category and further investigation of at-risk drinking within non-white ethnic groups is warranted.
Whilst being single was associated with being an at-risk drinker under the previous guidelines, this was no longer the case under the new guidelines. This may be accounted for by the greater increase in the percentage of at-risk drinkers in the reference category (married/cohabiting men: $13 \%$ increase in at-risk drinkers) compared to single men (12\% increase in at-risk drinkers). Whilst marriage is often associated with reduced drinking levels among men [60,61], this finding indicates that drinking habits of married men could be putting them at-risk. Whether the higher number of married/cohabiting men drinking between 14 and 21 units indicates an attempt to drink within (previous) guidelines would require further investigation.

Targeted screening and the offer of brief interventions for alcohol consumption have been implemented differently across the devolved nations. In Wales, clusters of health behaviours are targeted [39] and the finding that current and ex-smokers had more than twice higher odds of being at-risk drinkers under both the previous and new drinking guidelines (consistent with the literature on the common co-occurrence of smoking and alcohol consumption [62, 63]), indicates that England could benefit from a similar system. In Scotland, there has been a stronger focus on screening and intervention in line with a more severe alcohol problem [36], providing evidence of the benefits of tailoring services according to geographical location. The change in guidelines strengthened the association between living in the North East and North West and being an at-risk drinker and under the new guidelines only, living in the West Midlands, and London (when adjusted for ethnicity), were also significantly associated with being an at-risk drinker. Therefore more tailored service provision of screening and interventions for alcohol could be put in place in the North East, North West, West Midlands and London, where men have odds between one and a half and twice higher of being at-risk drinkers.

\section{Strengths, limitations and future research}

HSE is a nationally representative survey with consistently implemented alcohol measures, although it should be noted that HSE uses self-reported alcohol consumption measures and these have been found to underreport consumption [64], therefore the estimates in this paper may be conservative.

The current study sought to use retrospective data to explore the characteristics of men who would be considered at-risk from their drinking under the previous guidelines vs those considered at-risk under the new guidelines and therefore data from one year were used. Future research might use similar variables to explore cross-sectional changes over time to cover the period before and after the change to the guidelines. 


\section{Conclusions}

The change to the Low Risk Drinking Guidelines would have resulted in more than 2 million additional male atrisk drinkers in England. Men aged 55-74, current or ex-smokers, men in managerial or professional occupations and white men had greater odds of being at-risk drinkers under the new and previous guidelines and these are the groups already known to be drinking the most, strengthening the case for targeted screening and education around alcohol consumption for known groups of at-risk men. In addition, this study found that men in the West Midlands and London had greater odds of being at-risk drinkers under the new guidelines, and 16-35 year olds and married men had a marked increase in the proportion of at-risk drinkers. These are all groups that have not previously been identified as at-risk drinkers, and may benefit from specific education around the new Low Risk Drinking Guidelines.

\section{Abbreviations}

HSE: Health Survey for England; NatCen: National Centre for Social Research; NICE: National Institute for Health and Care Excellence; NS-SEC: National Statistics Socio-Economic Classification; SES: Socio-Economic Status

\section{Acknowledgements}

We are grateful to the participants in the HSE and the field staff and colleagues at the National Centre for Social Research (NatCen).

\section{Authors' contributions}

PC, LNF and NS all made substantial contributions to the conception, design, analysis and interpretation of data. PC drafted and LNF and NS revised and gave critical feedback on the manuscript. All authors gave final approval of the manuscript to be published.

\section{Funding}

The Health Survey for England is funded by NHS Digital (formerly the Health and Social Care Information Centre). PC is funded by a Medical Research Council Doctoral Training Programme, but this specific study was not funded. NHS Digital and the Medical Research Council had no role in the decision to undertake or publish this study, and no role in the conduct of this study.

\section{Availability of data and materials}

The datasets analysed within the current study are available from the UK Data Service: https://discover.ukdataservice.ac.uk/series/?sn=2000021

\section{Ethics approval and consent to participate}

Ethical approval details for each year of the survey used are listed below: 2015: West London Research Ethics Committee (reference number 14/LO/ 0862).

2014: Oxford A Research Ethics Committee (reference no. 12/SC/0317). 2013: Oxford A Research Ethics Committee (reference number 12/SC/0317). 2012: Oxford A Research Ethics Committee (reference number 10/H0604/56). 2011: Oxford A Research Ethics Committee (reference number 10/H0604/56). All participants consented to take part in Health Survey for England. Verbal consent was obtained for interviews and self-completion booklets (through which data for this study were collected) and the following measurements: height, weight, waist, hip, and blood pressure. Verbal consent was deemed sufficient for these aspects of the survey and responding to questions was considered to be consent. Participants were able to choose not to respond to specific questions or measurements. The use of verbal consent was approved by the Research Ethics Committee for each year of data collection. Written consent was obtained for blood samples, sending results to the GP and data linkage to Hospital Episode Statistics and the central register for mortality and cancer. Full details of the consent process are described elsewhere [65]. No further ethical approval was required for this study.
Consent for publication

Not applicable.

\section{Competing interests}

The authors declare that they have no competing interests.

Received: 31 May 2018 Accepted: 26 June 2019

Published online: 08 July 2019

\section{References}

1. Anderson P, Chisholm D, Fuhr DC. Effectiveness and cost-effectiveness of policies and programmes to reduce the harm caused by alcohol. Lancet. 2009:373:2234-46

2. Burton R, Henn C, Lavoie D, O'Connor R, Perkins C, Sweeney K, et al. A rapid evidence review of the effectiveness and cost-effectiveness of alcohol control policies: an English perspective. Lancet. 2017;389:1558-80. https:// doi.org/10.1016/S0140-6736(16)32420-5.

3. Department of Health. UK Chief Medical Officers' Low Risk Drinking Guidelines. 2016; August:11. https://www.gov.uk/government/uploads/ system/uploads/attachment_data/file/545937/UK_CMOs_report.pdf.

4. Nice. Alcohol-use disorders: Prevention. Public Heal Guid. 2010; June. http:// www.nice.org.uk/guidance/ $\mathrm{PH} 24$.

5. WHO. Global status report on alcohol and health. World Heal Organ 2014;: 1-100. doi:/entity/substance_abuse/publications/global_alcohol_report/en/ index.html.

6. Public Health England. Local alcohol profiles for England. 2017. https:// fingertips.phe.org.uk/profile/local-alcohol-profiles/data\#page/11/gid/ 1938132984/pat/6/par/E12000004/ati/102/are/E06000015/iid/91380/age/1/ sex/4. Accessed 5 Jan 2018.

7. NatCen Social Research. Health survey for England 2015 trend tables commentary. 2016. https:/digital.nhs.uk/data-and-information/publications/ statistical/health-survey-for-england/health-survey-for-england-2015-trend-tables.

8. NHS Digital. Statistics on Alcohol: England, 2017. 2017; May. http://digital. nhs.uk/catalogue/PUB23940. Accessed 2 Jan 2018.

9. McMurran M. Individual-level interventions for alcohol-related violence: expanding targets for inclusion in treatment programs. J Crim Justice. 2013; 41:72-80. https://doi.org/10.1016/j.jcrimjus.2012.09.001.

10. Office for National Statistics. Overview of violent crime and sexual offences: findings from analyses based on the year ending march 2015 crime survey for England and Wales and crimes recorded by the police covering different aspects of violent crime. March. 2016;2015:12-3. https://doi.org/10.1895/ wormbook.1.17.

11. Laslett AM, Room R, Dietze P, Ferris J. Alcohol's involvement in recurrent child abuse and neglect cases. Addiction. 2012;107:1786-93.

12. Wolf JP, Freisthler B. Understanding the roles of context, frequency, and quantity of alcohol consumption in child physical abuse: risks for mothers and fathers. J Fam Violence. 2016;31:539-48.

13. Matano RA, Wanat SF, Westrup D, Koopman C, Whitsell SD. Prevalence of alcohol and drug use in a highly educated workforce. J Behav Health Serv Res. 2002;29:30-44. https://doi.org/10.1007/BF02287830.

14. Jones S, Casswell S, Zhang J-F. The economic costs of alcohol-related absenteeism and reduced productivity among the working population of New Zealand. Addiction. 1995:90:1455-61.

15. Thavorncharoensap M, Teerawattananon Y, Yothasamut J, Lertpitakpong C, Chaikledkaew U. The economic impact of alcohol consumption: a systematic review. Subst Abus Treat Prev Policy. 2009;4:1-11.

16. House of Commons Science and Technology Committee. Alcohol guidelines. 2012; January:8.

17. British Medical Association (BMA). Alcohol: guidelines on sensible drinking. 1995.

18. Royal Colleges of Physicians P and GP. Alcohol and the heart in perspective: sensible limits reaffirmed. London; 1995.

19. Department of Health. Sensible drinking: Report of an inter-departmental working group. 1995; December:89. http://webarchive.nationalarchives.gov. uk/20130107105354/http://www.dh.gov.uk/prod_consum_dh/groups/dh_ digitalassets/@dh/@en/documents/digitalasset/dh_4084702.pdf.

20. Department of Health. Alcohol Guidelines Review - Report from the Guidelines development group to the UK Chief Medical Officers. 2016; January:1-44.

21. Lovatt M, Eadie D, Meier PS, Li J, Bauld L, Hastings G, et al. Lay epidemiology and the interpretation of low-risk drinking guidelines by adults in the United Kingdom. Addiction. 2015;110:1912-9. 
22. Roberts M (BBC N. Daily alcohol limit "unhelpful." BBC News. 2009. http:// news.bbc.co.uk/1/hi/health/8172982.stm. Accessed 4 Jan 2018.

23. Edwards G. Sensible drinking. BMJ Br Med J. 1996;312.

24. Department of Health. New alcohol guidelines show increased risk of cancer. News Story. 2016. https://www.gov.uk/government/news/newalcohol-guidelines-show-increased-risk-of-cancer. Accessed 3 Jan 2018.

25. BBC. New alcohol guidelines: what you need to know. BBC news. 2016. www.bbc.co.uk/news/uk-35252650. Accessed 3 Jan 2018

26. Press Association. No safe alcohol limit, new guidelines to say. The Guardian. 2016. https://www.theguardian.com/society/2016/jan/01/no-safealcohol-limit-new-guidelines-to-say. Accessed 3 Jan 2018.

27. Jones L, Bellis MA. CMO Alcohol Guidelines Review. Liverpool; 2014. http:// www.cph.org.uk/wp-content/uploads/2016/01/LJMU_CMO-AlcoholGuidelines-Health-Review.pdf

28. Holmes J, Angus C, Buykx P, Ally A, Stone T, Meier P, et al. Mortality and morbidity risks from alcohol consumption in the UK: analyses using the Sheffield alcohol policy model (v.2.7) to inform the UK chief medical officers' review of the UK lower risk drinking guidelines final report. 2016. https://www.sheffield.ac.uk/polopoly_fs/1.538671!/file/Drinking_Guidelines_ Final_Report_Published.pdf.

29. International Alliance for Responsible Drinking. Policy review: National Drinking Guidelines. 2016. http://www.iard.org/wp-content/uploads/2016/ 04/Policy-Review-National-Drinking-Guidelines.pdf. Accessed 13 Jan 2018.

30. Casswell S. Why have guidelines at all? A critical perspective. Drug Alcohol Rev. 2012;31:151-2.

31. Lindsay J. Healthy living guidelines and the disconnect with everyday life. Crit Public Health. 2010;20:475-87.

32. Stevely AK, Buykx P, Brown J, Beard E, Michie S, Meier PS, et al. Exposure to revised drinking guidelines and "COM-B" determinants of behaviour change: descriptive analysis of a monthly cross-sectional survey in England. BMC Public Health. 2018;18(1):1-9.

33. Holmes J, Brown J, Meier P, Beard E, Michie S, Buykx P. Short-term effects of announcing revised lower risk national drinking guidelines on related awareness and knowledge: a trend analysis of monthly survey data in England. BMJ Open [Internet]. 2016;6(12):e013804. Available from: http:// bmjopen.bmj.com/lookup/doi/10.1136/bmjopen-2016-013804

34. Rosenberg G, Bauld L, Hooper L, Buykx P, Holmes J, Vohra J. New national alcohol guidelines in the UK: public awareness, understanding and behavioural intentions. J Public Health (Bangkok) [Internet]. 2017 Sep 18;18. Available from: https://doi.org/10.1093/pubmed/fdx126

35. Babor TF, Higgins-Biddle JC, Saunders JB, Monteiro MG. AUDIT the alcohol use disorders identification test. 2nd ed. Geneva: Department of Mental Health and Substance Dependence, World Health Organization; 2001.

36. Heather N. Can screening and brief intervention lead to population-level reductions in alcohol-related harm? Addict Sci Clin Pract. 2012;7(1):15.

37. Kaner FSE, Dickinson HO, Beyer FR, Campbell F, Schlesinger C, Heather N, et al. Effectiveness of brief alcohol interventions in primary care populations. Cochrane database Syst rev; 2009. https://doi.org/10.1002/14651858. CD004148.pub3.www.cochranelibrary.com.

38. Kaner E, Bland M, Cassidy P, Coulton S, Dale V, Deluca P, et al. Effectiveness of screening and brief alcohol intervention in primary care (SIPS trial): pragmatic cluster randomised controlled trial. Bmj. 2013;346 jan09 2:e85011. https://doi.org/10.1136/bmj.e8501.

39. Drug and Alcohol Findings. "My GP says I drink too much": screening and brief intervention [internet]. Drug \& Alcohol Findings: hot topic. 2017. Available from: https://findings.org.uk/PHP/dl. php?file=alc_Bl.hot\&s=ml\#link.

40. Rao R (Tony). Guidelines on safe alcohol drinking are probably about right. BMJ Br Med J. 2015;351 http://www.bmj.com/content/351/bmj.h5082.abstract.

41. NatCen Social Research U. Health Survey for England, 2015: quick guide to the survey. 2016. https://files.digital.nhs.uk/publicationimport/pub22xxx/ pub22610/hse2015-quick-g.pdf. Accessed 20 Nov 2017

42. NatCen Social Research U. Health Survey for England 2015 questionnaires and showcards. 2015. http://doc.ukdataservice.ac.uk/doc/8280/mrdoc/pdf/ 8280_hse_2015_questionnaires_showcards.pdf. Accessed 2 Nov 2017.

43. Brown Laura (NatCen Social Research). Health Survey for England 2015 adult alcohol consumption. 2016. https://files.digital.nhs.uk/publicationimport/ pub22xxx/pub22610/hse2015-adult-alc.pdf. Accessed 5 Jan 2018.

44. Beard E, Brown J, West R, Angus C, Brennan A, Holmes J, et al. Deconstructing the alcohol harm paradox: a population based survey of adults in England. PLoS One. 2016;11:1-17.
45. Lewer D, Meier P, Beard E, Boniface S, Kaner E. Unravelling the alcohol harm paradox: a population-based study of social gradients across very heavy drinking thresholds. BMC Public Health. 2016;16:1-11. https://doi.org/10. 1186/s12889-016-3265-9.

46. Dinescu D, Turkheimer E, Beam CR, Horn EE, Duncan G, Emery RE. Is marriage a buzzkill? A twin study of marital status and alcohol consumption. J Fam Psychol. 2016;30:698-707.

47. Power C, Rodgers B, Hope S. Heavy alcohol consumption and marital status: disentangling the relationship in a national study of young adults. Addiction. 1999;94:1477-87.

48. Castillo JM, Jivraj S, Ng Fat L. The regional geography of alcohol consumption in England: comparing drinking frequency and binge drinking. In: Heal Place; 2017;43 November 2015. p. 33-40. https://doi.org/ 10.1016/j.healthplace.2016.11.007.

49. Roberts SE, Williams JG, Meddings D, Goldacre MJ. Incidence and case fatality for acute pancreatitis in England: geographical variation, social deprivation, alcohol consumption and aetiology - a record linkage study. Aliment Pharmacol Ther. 2008;28:931-41.

50. Shelton N, Savell E. The geography of binge drinking: the role of alcoholrelated knowledge, behaviours and attitudes. Results from the health survey for England 2007. Heal Place. 2011;17:784-92. https://doi.org/10.1016/j. healthplace.2011.02.004.

51. Bécares L, Nazroo J, Stafford M. The ethnic density effect on alcohol use among ethnic minority people in the UK. J Epidemiol Community Health. 2011;65:20-5.

52. Bayley M, Hurcombe R. Drinking patterns and alcohol service provision for different ethnic groups in the UK: a review of the literature. Ethn Inequalities Heal Soc Care. 2011;3:6-17. https://doi.org/10.5042/eihsc.2011.0073.

53. Mawditt C, Sacker A, Britton A, Kelly Y, Cable N. The clustering of healthrelated behaviours in a British population sample: testing for cohort differences. Prev Med (Baltim). 2016;88:95-107. https://doi.org/10.1016/j. ypmed.2016.03.003.

54. Fat LN, Cable N, Marmot MG, Shelton N. Persistent long-standing illness and non-drinking over time, implications for the use of lifetime abstainers as a control group. J Epidemiol Community Health. 2014;68:71-7.

55. C a G, Freeborn DK, Polen MR. Gender and alcohol use: the roles of social support, chronic illness, and psychological well-being. J Behav Med. 2001;24:383-99.

56. NatCen Social Research U. Health survey for England 2015 trend tables population number estimates guide. 2016. https://files.digital.nhs.uk/ publicationimport/pub22xxx/pub22616/hse2015-pop-no-est-user-guide.pdf

57. Pittam $G$, Lines $C$. Screening for alcohol misuse in adults external review against programme appraisal criteria for the: UK National Screening Committee (UK NSC); 2016.

58. Drinkaware. Almost half of the UK's middle-aged men are drinking above the low risk weekly alcohol guidelines. 2016. https://www.drinkaware.co.uk/press/halfof-uk-middle-aged-men-drinking-above-guidelines/ Accessed 14 Feb 2019.

59. Angus, C. A generation of hidden drinkers: What's happening to the drinking of the over 50s? 2019. https://drinkwiseagewell.org.uk/ageneration-of-secret-drinkers-whats-happening-to-the-drinking-of-the-over50s/ Accessed 14 Feb 2019.

60. Curran P, Muthen B, Harford T. The influence of changes in marital status on developmental trajectories of alcohol use in young adults. Journal of Alcohol Studies. 1998;59:647-58.

61. Reczek C, Pudrovska T, Carre D, Thomeer M, Umberson D. Marital histories and heavy alcohol use among older adults. J Health Soc Behav. 2016:57:77-96.

62. Falk D, Yi H-Y, Hiller-Sturmhofel S. An epidemiologic analysis of co-occurring alcohol and tobacco use and disorders: findings from the National Epidemiologic Survey on alcohol and related conditions. Alcohol Res Health. 2006;29:162-71.

63. Beard E, West R, Michie S, Brown J. Association between smoking and alcohol-related behaviours: a time-series analysis of population trends in England. Addiction. 2017;112:1832-41.

64. Boniface S, Shelton N. How is alcohol consumption affected if we account for under-reporting? A hypothetical scenario. Eur J Pub Health. 2013;23: 1076-81.

65. NHS Digital. Health survey for England methods. 2016. Accessed at: http:// healthsurvey.hscic.gov.uk/media/37742/hse2015-methods.pdf. Accessed 20 Jun 2019.

\section{Publisher's Note}

Springer Nature remains neutral with regard to jurisdictional claims in published maps and institutional affiliations. 